\title{
MAGNETIC HELICITY OF OSCILLATING CORONAL LOOPS
}

\author{
V.Krishan ${ }^{1}$ and E.R.Priest ${ }^{2}$ \\ 1 Indian Institute of Astrophysics, Bangalore 560034, India \\ 2 Univ. of St. Andrews, Mathematical Institute, St.Andrews \\ KY 169 SS, Fife, Scotland
}

The dynamics of the velocity and magnetic field in a coronal loop is studied using ideal MHD equations and the Chandrasekhar-Kendall representation. The complete dynamics is described by a set of infinite, coupled nonlinear ordinary differential equations which are first-order in time for the expansion coefficients of the velocity and magnetic field. Here, the coronal loop plasma is represented by a superposition of the three [ $\mathrm{n}=0=\mathrm{m} ; \mathrm{n}=\mathrm{m}=1$ and $\mathrm{n}=\mathrm{m}=1$ ] lowest-order C-K functions. This system, when perturbed linearly from its equilibrium state exhibits sinusoidal oscillations. The frequency $S$ of these oscillations is given by (Krishan et al 1988):

$$
S^{2}=A \eta_{a}^{2}+B \eta_{b}^{2}+C \eta_{c}^{2}
$$

where $A, B$ and $C$ are constants and $\eta$ 's are the equilibrium amplitudes of velocity field which are also equal to magnetic field amplitudes. The three quadratic invariants of this system are

the total energy $E=2\left[\lambda_{a}^{2} \eta_{a}^{2}+\lambda_{b}^{2} \eta_{b}^{2}+\lambda_{c}^{2} \eta_{c}^{2}\right]$

the magnetic Helicity $H_{m}=\lambda_{a} \eta_{a}^{2}+\lambda_{b} \eta_{b}^{2}+\lambda_{c} \eta_{c}^{2}$

and the cross helicity $\mathrm{H}$ becomes equal to the total energy under the conditions of equilibrium $\vec{V}=\vec{B}$, which is an aligned Alfvenic state. $\lambda$ 's are the characteristic wave-vectors of the three modes. From equations (1) and (2), we found that the frequency $S$ can be expressed in a very simple form as

$$
S^{2}=\frac{A H_{m}}{\lambda_{a}}
$$

Here $\lambda_{a}$, where $a \equiv(0,0)$ mode, can be expressed in terms of the ratio of poloidal $\psi_{p}$ to toroidal $\psi_{t}$ magnetic flux; $\lambda_{b}$ and $\lambda_{c}$ are numerical values

E. R. Priest and V. Krishan (eds.), Basic Plasma Processes on the Sun, 256-257.

C 1990 IAU. Printed in the Netherlands. 
related to zeros of Bessel functions (Montgomery et al (1978)) obtained from

$$
\begin{aligned}
& \left(\psi_{t} / \psi_{p}\right)=-\frac{R}{L} \frac{\left|\lambda_{a}\right|}{\lambda_{a}} \frac{J_{0}^{\prime}\left(\lambda_{a} R\right)}{J_{0}\left(\lambda_{a} R\right)} \\
& \frac{2 \pi R}{L} \gamma_{b} J_{1}^{\prime}\left(\gamma_{b} R\right)+\lambda_{b} J_{1}\left(\gamma_{b} R\right)=0
\end{aligned}
$$

and $\frac{2 \pi R}{L} \gamma_{c} J_{-1}^{\prime}\left(\gamma_{c} R\right)+\lambda_{c} J_{-1}\left(\gamma_{c} R\right)=0$

where $\lambda=\left[\gamma^{2}+(2 \pi R)^{2} / L^{2}\right]^{1 / 2}$

and $(L, R)$ are the length and radius of the cylindrical plasma loop. By measuring the periods of oscillating loop prominences often observed in coronograph movies, one has now a way of estimating magnetic helicity which eludes any direct measurement.

\section{References}

Krishan,V. Berger,M. and Priest,E.R., 1988 in "Solar and Stellar Coronal Structure and Dynamics" proceedings of the ninth Sacramento peak summer symposium Aug. 1987.

Montgomery,D., Turner,L. and Vahala G. : 1978 Phys. Fluids $\underline{21,} 757$. 